\title{
Constants of Motion in Local Field Theory (Coleman's Theorem Revisited)
}

\author{
J. Bros \\ CEN Saclay, Gif-sur-Yvette, France \\ D. Buchholz \\ II. Institut für Theoretische Physik, Universität Hamburg, D-2000 Hamburg 50, \\ Federal Republik of Germany \\ V. Glaser \\ CERN, Geneva, Switzerland
}

\begin{abstract}
We analyze the space integrals $Q=\int d^{3} x \varrho(\boldsymbol{x})$ of finitely localized densities $\varrho$. It turns out that the time translated operators $Q(t)$ are polynomials in $t$ if $Q$ annihilates the vacuum. In particular, $Q(t)=Q$ in models with shortrange forces and complete particle interpretation. These results are valid in the Haag-Araki framework of field theory as well as in the Wightman formalism. Lorentz covariance is not needed in the proofs.
\end{abstract}

\section{Introduction and Main Results}

In a recent paper Gal-Ezer and Reeh have shown that the space-integral of the zeroth component of a tensor current defines a conserved charge if it annihilates the vacuum [1]. This work generalizes a result which is known in the literature as Coleman's theorem [2] and it adds to a series of papers which were stimulated by Coleman's original article. (See for example [3] and the references therein.) Although there may be differences in style and rigour the idea of proof in all these investigations is essentially the same. The argument is based on a detailed study of the two-point function of the divergence of the current. It follows from Lorentz-covariance that the intermediate states which contribute to this function have zero mass if the charge annihilates the vacuum. Thus the two-point function vanishes in theories with a mass gap and the current is conserved. In the presence of massless particles one cannot conclude quite as much. There the recent result of Gal-Ezer and Reeh is the best one to be expected.

Since Lorentz-covariance is very essential for the above argument one may ask whether Coleman's theorem depends crucially on this assumption and it is the aim of the present paper to clarify this point. We shall show that locality and spectrum condition are already sufficient ingredients for a proof and that Lorentzcovariance is not needed. Moreover, in an appropriate formulation Coleman's theorem holds also for finitely localized quantities like Haag-Araki fields or locally smeared polynomials in the basic Wightman-fields. It is in the latter case in which we can formulate the most easily comprehensible version of our main 
result. To specify the framework let us assume that we are dealing with a WigthmanTheory of one real field $\phi$, which satisfies the usual postulates [4] with the possible exception of Lorentz-covariance. We denote the vacuum vector by $\Omega$ and the unitary representation of translations by $(t, x) \rightarrow T(t, x)$. We then have the

Theorem. If $\varrho$ is a locally smeared polynomial in the basic field $\phi$ and if

$$
\int d^{3} x(\Omega,[\varrho(x), A] \Omega)=0
$$

for all locally smeared polynomials $A$ in $\phi$, then there exists a closable operator $Q$ defined on $D(Q)=\{A \Omega\}$ by

$$
Q \cdot A \Omega=\int d^{3} x[\varrho(\boldsymbol{x}), A] \Omega
$$

such that on $D(Q)$

$$
\left(d^{N} / d t^{N}\right) T(t) Q T(t)^{-1}=0
$$

for some finite $N$. If the theory has a mass-gap and is asymptotically complete, then $N=1$.

Thus at least in theories with short-range forces and a complete particle interpretation, $Q$ is a constant of motion. It is hard to believe that there exist models in which relation (3) does not hold for $N=1$. However we want to emphasize, that the existence of an operator $Q$ which satisfied (3) for some $N>1$ would not be a disaster but - on the contrary - most welcome. In the case $N=2$, for example, $Q_{1}=(d / d t) T(t) Q T(t)^{-1}$ would be a conserved quantity and $Q_{2}=T(t) Q T(t)^{-1}-t \cdot Q_{1}$ would not depend on $t$. Hence $Q_{2}$ would be the generator of a local symmetry transformation (similar to the generators of Lorentz-boosts) and we would have obtained two symmetries from one density $\varrho$. But this is probably too much to hope.

The first and most important step in the proof of the theorem consists of showing that

$$
\int d^{3} x\left(\Omega, A^{\prime}[\varrho(t, x), A] \Omega\right)=P(t)
$$

where $P(t)$ is some polynomial in $t$ and $A, A^{\prime}$ are local Wightman-fields. To verify this equation we have to go through a rather tedious analysis of the analytic properties of the three-point function generated by $A, A^{\prime}$, and $\varrho$. We shall exploit the fact that the left-hand side of (4) can be calculated from the double discontinuities of this function and that relation (1) together with the Jacobi identities allow a considerable enlargement of its domain of regularity. (Positivity of the metric is nowhere needed in this part of the argument and relation (4) therefore also holds in models with indefinite metric.) In the remainder of the proof we shall then infer from (4) the statement of the theorem using arguments from operator theory.

\section{The Key Lemma}

This section is devoted to the statement that the matrix elements (4) are polynomials in $t$. 
We shall use the following notation. Let $A_{1}(x)=T(x) \varrho T(x)^{-1}$ and $A_{i}(x)=$ $T(x) A_{i} T(x)^{-1}, i=2,3$, where $A_{i}$ are two fixed compactly smeared Wightman polynomials in the basic fields. The truncated Wightman function

$$
\left(\underline{\Omega}, A_{i}\left(x_{i}\right) A_{j}\left(x_{j}\right) A_{k}\left(x_{k}\right) \Omega\right)^{T},
$$

where $(i, j, k)$ is a permutation of $(1,2,3)$, will be denoted by $\left\langle x_{i} x_{j} x_{k}\right\rangle$. It is a $C^{\infty}$ function of the coordinate differences polynomially bounded at infinity. Its Fourier transform will be denoted by $\delta_{4}\left(p_{1}+p_{2}+p_{3}\right)\langle i j k\rangle$. $\langle i j k\rangle$ can be regarded as a tempered distribution depending on any two of the three 4-vectors $p_{l}$ linked by the relation $p_{1}+p_{2}+p_{3}=0$. Its support is contained in $\left\{p_{i} \in \bar{V}_{+}\left(m_{i}\right), p_{k} \in \bar{V}_{-}\left(m_{k}\right)\right\}$, where $m_{l}$ are the threshold masses.

We are interested in the integrals $\int\left\langle x_{i} x_{j} x_{k}\right\rangle d^{3} x_{1}$, the Fourier transform of which are formally given by the restriction of $\langle i j k\rangle$ to $p_{1}=\mathbf{0}$ and we shall use the following type of notation: $\langle 3 \hat{1} 2\rangle=\left.\langle 312\rangle\right|_{\boldsymbol{p}_{1}=0}, \hat{p}_{1}=\left(p_{1}^{0}, \mathbf{0}\right)$. This restriction gives rise to well defined tempered distributions in the case of theories with strictly positive minimal mass, because locality and spectrum imply that the momentum space Wightman functions have a $C^{\infty}$ dependence on the space components of the 4-vectors $p_{l}$. In a 0 -mass theory these expressions might be meaningless. But according to (1) and (4) it suffices for our purposes to consider the expressions $\langle j[\hat{1}, k]\rangle,\langle[\hat{1}, k] j\rangle$ and $\langle[\hat{1}, j k]\rangle$. These are always meaningful. The invariance condition of the vacuum (1) reads in momentum space

$$
\langle[\hat{1}, j k]\rangle=0, \quad(j, k)=(2,3) \text { or }(3,2)
$$

and it is convenient to define in view of (5) and the Jacobi identity

$$
\langle j \hat{1} k\rangle \equiv\langle j[\hat{1}, k]\rangle=\langle[j, \hat{1}] k\rangle \text {. }
$$

The spectral properties of the Wightman functions imply then

$$
\operatorname{supp}\langle j \hat{1} k\rangle \subset\left\{\hat{p}_{1}+p_{2}+p_{3}=0, p_{j} \in \bar{V}_{+}, p_{k} \in \bar{V}_{-}\right\} \text {. }
$$

In a massive theory the new definition (6) agrees of course with the old one.

The content of this section is the following.

Lemma 1. The linear properties of the 3-point function and condition (5) imply $\operatorname{supp}\langle 3 \hat{1} 2\rangle \subset\left\{\left(p_{1}^{0}, p_{2}\right): p_{1}^{0}=0\right\}$. Because of the spectral condition (7) this tempered distribution is of the form

$$
\langle 3 \hat{1} 2\rangle=\sum_{n=0}^{N} \delta^{(n)}\left(p_{1}^{0}\right) f_{n}\left(p_{2}\right)
$$

for some finite $N$ where $f_{n} \in \mathscr{S}^{\prime}\left(\mathbb{R}^{4}\right)$ with $\operatorname{supp} f_{n} \in \bar{V}_{-}(m), n=0,1, \ldots, N$ and $m=\max \left(m_{1}, m_{2}\right)$.

It is clear that this Lemma implies formula (4) of the Introduction. It is also clear that we may ignore the mass thresholds in the proof, i.e. put all $m_{l}=0$.

Proof. The idea of the proof is to use the fact that the double commutators $\langle[i,[j, k]]\rangle$ are the double discontinuities of the momentum space Green's function $H(k)$ analytic in the well-known Källen-Wightman domain $\mathscr{D}[5]^{1}$. Here $k=\left(k_{1}, k_{2}, k_{3}\right)$,

\footnotetext{
1 No explicit Lorentz covariance is actually needed in the derivation of this domain. This follows from the Glaser-Streater Theorem [6]
} 
$k_{1}+k_{2}+k_{3}=0$, where $k_{l}=p_{l}+i q_{l}, l=1,2,3$. Let us denote by $\hat{H}$ the restriction of $H$ to the 5-dimensional complex linear subspace $k_{1}=0$. This function is analytic in the domain $\hat{\mathscr{D}}=\mathscr{D} \cap\left\{\boldsymbol{k}_{1}=0\right\}$; its boundary values at the real points of $\hat{\mathscr{D}}$ exist according to general theorems on functions analytic in tubes (compare e.g. [7]); its double discontinuities are the commutator vacuum expectation values $\langle[i,[\hat{1}, j]]\rangle$ e.c.t. we are interested in. We are therefore faced with a generalized Edge-of-the-Wedge problem, in which the real region of coincidence is very large as a consequence of the condition (5). Now, in an E.o.W. problem a given real region of coincidence can in general be enlarged by the use of the "Double cone" and "Re-entrant Nose" theorems. This phenomenon was first discovered by Dyson [8] in a special case; a fairly general treatment can be found e.g. in [9]. In the problem at hand it turns out that an appropriate version of the Re-entrant Nose Theorem shrinks the support of the matrix elements $\langle j \hat{1} k\rangle$ given a priori by (7) to that given by the Lemma.

We shall find that only a partial analytic completion of the primitive 3-point function domain is needed for our purposes. This completion can be obtained e.g. by the application of the Jost-Lehmann-Dyson formula to the commutator functions $\left\langle\left[x_{2},\left(x_{3}, x_{1}\right)_{ \pm}\right]\right\rangle$for fixed $x_{3}-x_{1}$. Here $\left(x_{3}, x_{1}\right)_{ \pm}=\Theta_{ \pm}\left(x_{3}^{0}-x_{1}^{0}\right) \times$ $\left[A_{3}\left(x_{3}\right), A_{1}\left(x_{1}\right)\right]$ are the retarded respectively advanced commutators, (the multiplication by the step functions is legitimate because the $x$-space Wightman functions are $C^{\infty}$ in view of the smearing of $A_{2,3}$ ). Taking into account the support properties in configuration and momentum space with respect to $x_{2}-x_{1}$ one gets by a slight generalization of [10]

$$
\left\langle\left[x_{2},\left(x_{3}, x_{1}\right)_{ \pm}\right]\right\rangle=\int \varphi_{ \pm}\left(x_{2}^{\prime}-x_{1}, x_{2}^{\prime}-x_{3}, \kappa^{2}\right) \Delta\left(x_{2}-x_{2}^{\prime}, \kappa^{2}\right) d^{4} x_{2}^{\prime} d \kappa^{2} .
$$

Here $\varphi_{ \pm}\left(x, y, \kappa^{2}\right) \in \mathscr{S}^{\prime}\left(\mathbb{R}^{9}\right)$ with $\operatorname{supp} \varphi_{ \pm} \subset\left\{x \in \bar{V}_{ \pm} \mp a, y \in \bar{V}_{\mp} \pm a, \kappa^{2} \geqq 0\right\}$, where $a=\left(a_{0}, 0\right)$ is a positive time-like vector with $a_{0}$ sufficiently large to swallow the finite localization of the fields. In momentum space this gives

$$
\left\langle\left[2,(3,1)_{ \pm}\right]\right\rangle=\tilde{\varphi}_{ \pm}\left(p_{1}, p_{3}, p_{2}^{2}\right) \varepsilon\left(p_{2}^{0}\right) \equiv G_{ \pm}\left(p_{2}, p_{3}\right) \varepsilon\left(p_{2}^{0}\right),
$$

where $\tilde{\varphi}_{ \pm}\left(p_{1}, p_{3}, \kappa^{2}\right) \in \mathscr{S}^{\prime}\left(\mathbb{R}^{9}\right)$ are the Fourier transforms of $\varphi_{ \pm}$with respect to $x$ and $y$; they are boundary values of functions $\tilde{\varphi}_{ \pm}\left(k_{1}, k_{3}, \kappa^{2}\right)$ analytic in the tubes $\mathscr{T}^{ \pm}=\left\{\operatorname{Im} k_{1} \in V_{ \pm}, \operatorname{Im} k_{3} \in V_{\mp}\right\}$ with values in tempered distributions with respect to $\kappa^{2}$. Thus the "simple discontinuities" $G_{ \pm}\left(p_{2}, p_{3}\right)$ of the Green's function $H$ (according to our conventions $p_{1}$ is defined by the relation $p_{1}+p_{2}+p_{3}=0$ ) are boundary values of functions $G_{ \pm}\left(k_{2}, k_{3}\right)$ analytic in $\mathscr{T}_{\varrho}^{ \pm}=\mathscr{T}^{ \pm} \cap \mathscr{H}_{\varrho}$ for each fixed $\varrho \geqq 0$, where $\mathscr{H}_{\varrho}$ is the complex hyperboloid $\left\{k_{2}: k_{2}^{2}=\varrho\right\}$ and $k_{1}+k_{2}+k_{3}=0$. Thus $\mathscr{T}_{\varrho}^{ \pm}$are domains on the complex analytic mainfold $\mathscr{M}_{\varrho}=\mathbb{C}_{k_{3}}^{4} \times \mathscr{H}_{\varrho}$ of complex dimension 7, and it is easily checked that the real points of $\mathscr{M}_{\varrho}$, viz.

$$
\mathbb{R}_{p_{3}}^{4} \times\left\{p_{2}^{2}=\varrho, p_{2}^{0}>0\right\} \quad \text { and } \quad \mathbb{R}_{p_{3}}^{4} \times\left\{p_{2}^{2}=\varrho, p_{2}^{0}<0\right\},
$$

are boundary points of $\mathscr{T}_{\varrho}^{+}\left(\mathscr{T}_{\varrho}^{-}\right)$that can be connected by a "crossing" path contained in $\mathscr{T}_{\varrho}^{+}\left(\mathscr{T}_{\varrho}^{-}\right)$.

For a different derivation of these results compare [11]. Let us also note that the fixing of the parameter $\varrho$ in the above reasoning is not completely rigorous in view of the distribution character of the function in question. We mention as an aside that the analytic functions $G_{ \pm}$are actually measures with respect to 
the variable $\varrho$ as a consequence of the positivity of the metric of the underlying Hilbert space. However, using e.g. a version of the general regularization procedure expounded in [12] we may proceed as if the functions in question were continuous.

We restrict now our attention to the 4-dimensional complex manifold $\hat{\mathscr{M}}_{\varrho}=\mathscr{M}_{\varrho} \cap\left\{\boldsymbol{k}_{1}=0\right\}$ and denote by $\hat{G}_{ \pm}$and $\hat{\mathscr{T}}_{\varrho}^{ \pm}$the restrictions of $G_{ \pm}$and $\mathscr{T}^{ \pm}$ to $\hat{\mathscr{M}}_{\varrho}$. Since (6) implies

$$
\left\langle\left[(3, \hat{1})_{+}, 2\right]\right\rangle-\left\langle\left[(3, \hat{1})_{-}, 2\right]\right\rangle=\langle 3 \hat{1} 2\rangle+\langle 2 \hat{1} 3\rangle
$$

we are faced with the Edge-of-the-Wedge problem $\hat{\mathscr{T}}_{\varrho}^{+} \cup \hat{\mathscr{T}}_{\varrho}^{-} \cup \hat{\mathscr{R}}_{\varrho}$ on the manifold $\hat{\mathscr{M}}_{\varrho}$, where $\hat{\mathscr{R}}_{\varrho}$ are the real coincidence points of the pair of analytic functions $\hat{G}_{ \pm}$given according to (7) by

$$
\hat{\mathscr{R}}_{\varrho}=\mathcal{C}\left(\mathscr{C}^{+} \cup \mathscr{C}^{-}\right) \cap \hat{\mathscr{M}}_{\varrho}, \quad \mathscr{C}^{ \pm}=\left\{p_{3} \in \bar{V}_{ \pm}, p_{2} \in \bar{V}_{\mp}\right\} .
$$

By writing $k_{2}=\left( \pm \sqrt{\boldsymbol{k}_{3}^{2}+\varrho},-\boldsymbol{k}_{3}\right)$ we obtain a parametrization of $\hat{\mathscr{M}}_{\varrho}$ by the 4-vector $k_{3}$. This parametrization is regular everywhere except at the points of the critical manifold $K_{\varrho}=\left\{k_{3} \in \mathbb{C}^{4}: \boldsymbol{k}_{3}^{2}+\varrho=0\right\}$. If we denote by $\pi$ the projection of $\hat{\mathscr{M}}_{\varrho}$ on $\mathbb{C}_{k_{3}}^{4}$, the above parametrization can be regarded as the inverse map

$$
k_{3} \stackrel{\pi^{-1}}{\longrightarrow}\left\{\begin{array}{l}
k_{2}=\left( \pm \sqrt{\boldsymbol{k}_{3}^{2}+\varrho},-\boldsymbol{k}_{3}\right) \\
k_{3}
\end{array}\right.
$$

which means that $\pi$ is two to one. We then put $F_{ \pm}\left(k_{3}\right)=\hat{G}_{ \pm}\left(\pi^{-1} k_{3}\right), F_{ \pm}$being double valued analytic functions over $\pi(\hat{\mathscr{T}} \stackrel{ \pm}{ \pm}) \backslash K_{\varrho}$.

We now check that $F_{ \pm}$are analytic in $T^{\mp} \backslash K_{\varrho}$, where $T^{ \pm}=\left\{k_{3} \in \mathbb{C}^{4}: \operatorname{Im} k_{3} \in V^{ \pm}\right\}$ are the usual forward respectively backward tubes. To this end it is necessary to show that $\pi\left(\hat{\mathscr{T}}_{\varrho}^{ \pm}\right)=T^{\mp}$, an elementary exercise left to the reader. The two determinations of $F_{ \pm}$correspond near the real points to the two boundary values $\varepsilon\left(p_{2}^{0}\right)= \pm 1$ in formula (8). Let us denote them by $F_{ \pm}^{\varepsilon}, \varepsilon= \pm$. Setting $\Delta F^{\varepsilon}=F_{+}^{\varepsilon}-F_{-}^{\varepsilon}$ for the discontinuity on the reals, we get from (7), (8), and (9) $\Delta F^{-}=\langle 3 \hat{1} 2\rangle$ so that supp $\Delta F^{-} \subset \bar{V}_{+}$; similarly $\Delta F^{+}=-\langle 2 \hat{1} 3\rangle$ and $\operatorname{supp} \Delta F^{+} \subset \bar{V}_{-}$. Now, since $\operatorname{Re}\left(\boldsymbol{k}_{3}^{2}+\varrho\right)>0$ in the tubes $T_{\varrho}^{ \pm}=T^{ \pm} \cap\left\{\left(\operatorname{Im} \boldsymbol{k}_{3}\right)^{2}<\varrho\right\}$, the determinations $F_{ \pm}^{\varepsilon}$ are single-valued in these domains, so that $F_{+}^{-}$and $F_{-}^{-}$are restrictions to $T_{\varrho}^{+}$and $T_{\varrho}^{-}$of a function $F^{-}$analytic in $H^{-}=H\left(T_{\varrho}^{+} \cup T_{\varrho}^{-} \cup C \bar{V}_{+}\right)=$the envelope of holomorphy of the E.o.W. problem $T_{\varrho}^{+} \cup T_{\varrho}^{-} \cup \mathcal{C} \bar{V}_{+}$. Here $C \bar{V}_{+}$are the real points of coincidence $\left\{p_{3}^{0}<\left|\boldsymbol{p}_{3}\right|\right\}$; they are interior points of $H^{-}$by the local E.o.W. theorem. A similar statement holds for the pair of analytic functions $F_{+}^{+}$and $F_{-}^{+}$.

If in the above E.o.W. problem the tubes $T_{\varrho}^{ \pm}$could be replaced by the forward and backward tubes $T^{ \pm}$(i.e. if the double valuedness of the functions $F_{ \pm}$in the domains $T^{ \pm} \backslash K_{\varrho}$ could be neglected), the Re-entrant Nose Theorem $([8,9])$ would immediately yield $H^{\mp}=\mathbb{C}^{4}$, i.e. $\operatorname{supp} \Delta F^{ \pm}$is the empty set. What we shall actually show is that the region of analyticity of $F^{-}$can be extended to the real points $\left\{p_{3}^{0}<\sqrt{\boldsymbol{p}_{3}^{2}+\varrho}\right\}$, which means that

$$
\operatorname{supp} \Delta F^{-} \subset\left\{p_{3} \in \mathbb{R}^{4}: p_{3}^{0} \geqq \sqrt{\boldsymbol{p}_{3}^{2}+\varrho}\right\} \text {. }
$$

By symmetry we shall then also have $\operatorname{supp} \Delta F^{+} \subset\left\{p_{3}^{0} \leqq-\sqrt{\boldsymbol{p}_{3}^{2}+\varrho}\right\}$. Since $p_{2}^{2}=\varrho$ was an arbitrary non-negative number, these support properties are equivalent to

$$
\begin{aligned}
& \operatorname{supp}\langle 3 \hat{1} 2\rangle \subset\left\{p_{3} \in \bar{V}_{+}, p_{2} \in \bar{V}_{-}, p_{2}+p_{3}=0, p_{2}^{0}+p_{3}^{0} \geqq 0\right\} \\
& \operatorname{supp}\langle 2 \hat{1} 3\rangle \subset\left\{p_{3} \in \bar{V}_{-}, p_{2} \in \bar{V}_{+}, p_{2}+p_{3}=0, p_{2}^{0}+p_{3}^{0} \leqq 0\right\} .
\end{aligned}
$$


Now the whole argument leading from (8) to (11) is valid also with the variables 2 and 3 interchanged. From this follows the statement of the Lemma.

We are therefore left with the proof of (10). To this end we shall first enlarge the analyticity domain of the double valued functions $F_{\delta}, \delta= \pm$. We can always write

$$
F_{\delta}\left(k_{3}\right)=F_{\delta}^{s}\left(k_{3}\right)+\left(k_{3}^{2}+\varrho\right)^{-1 / 2} F_{\delta}^{a}\left(k_{3}\right)
$$

where

$$
F_{\delta}^{S}=\frac{1}{2}\left(F_{\delta}^{+}+F_{\delta}^{-}\right)
$$

and

$$
F_{\delta}^{a}=\frac{1}{2}\left(F_{\delta}^{+}-F_{\delta}^{-}\right) \cdot\left(\boldsymbol{k}_{3}^{2}+\varrho\right)^{1 / 2}
$$

are analytic and single-valued in $T^{\delta} \backslash K_{\varrho}$; they are analytic and single-valued even in the whole of $T^{\delta}$ because by their very definition they are bounded at the points of the critical analytic manifold $K_{\varrho}$. Besides by our previous discussion the pair of functions $F_{ \pm}^{s}$ as well as the pair of functions $F_{ \pm}^{a}$ coincide at the real points $\left\{p_{3}^{2}<0\right\}$. As it is well known, the envelope of holomorphy of this E.o.W. problem is the complement of the positive cut $D=\left\{k_{3} \in \mathbb{C}^{4}: k_{3}^{2} \neq \sigma \geqq 0\right\}$. This implies that the pair of functions $F_{ \pm}$are two branches of a single function $F$ analytic and double valued in the domain $D \backslash K_{\varrho}$.

Let us now concentrate our attention to the determination $F^{-}$of $F$ which was previously shown to be analytic in a complex neighbourhood $N_{+}$of the real points $C \bar{V}_{+}$. In order to prove (10) let us first treat the case of a theory with a strictly positive minimal mass $m$. In such a theory the sets $D, C \bar{V}_{+}, N_{+}$are replaced by $\mathrm{D}_{m}=\left\{k_{3}^{2} \neq \sigma \geqq m^{2}\right\}, C \bar{V}_{+}^{m}$ and $N_{+}^{m}$. Because of rotational symmetry we can restrict ourselves to the two-dimensional complex plane $k_{3}=\left(k_{3}^{0}, k_{3}^{1}, 0,0\right)$. We then cut the domain $\left(D_{m} \backslash K_{\varrho}\right) \cup N_{+}^{m}$ by a family of hyperbolae of the form

$$
H_{a, \alpha}=\left\{\left(k_{3}^{0}+a\right)^{2}-\left(k_{3}^{1}\right)^{2}=\alpha\right\}
$$

with the real parameters $a$ and $\alpha$ varying in the intervals $0 \leqq a \leqq a_{0}, 0<\alpha<\varrho$, and apply the disk theorem, noticing that the intersections of the singular sets $S_{m}=\left\{k_{3}: k_{3}^{2}=\sigma \geqq m^{2}\right\}$ and $K_{\varrho}$ by any hyperbola $H_{a, \alpha}$ remain disjoint when $a$ and $\alpha$ vary in their intervals; (a branch cut starting from $K_{\varrho}$, which defines the determination $F^{-}$will also remain disjoint from $S_{m}$ ). The situation can be visualized in the complex plane of the angular variable $\psi$

$$
H_{a, \alpha}=\left\{k_{3}^{0}+a=\sqrt{\alpha} \operatorname{ch} \psi, k_{3}^{1}=\sqrt{\alpha} \operatorname{sh} \psi\right\}
$$

or alternatively in the complex $\lambda$-plane, if we use the parametrization $2 \operatorname{ch} \psi=$ $\lambda+\lambda^{-1}, 2 \operatorname{sh} \psi=\lambda-\lambda^{-1}$. When $a$ increases from $0, H_{a, \alpha} \cap\left(S_{m} \backslash N_{+}^{m}\right)$ is empty at the beginning (for $\alpha=\alpha_{0}$ small enough) because of $m>0$. Now keeping $a=a_{0}$ fixed and increasing $\alpha$ from $\alpha_{0}$ to $\varrho$ one finds that there appear compact curves of $H_{a, \alpha} \cap\left(S_{m} \backslash N_{+}^{m}\right)$ on which $F^{-}$was not known to be analytic, and which can be removed in view of the Disk theorem. In particular one thus obtains analyticity at all real points of the form

$$
\left\{p_{3}^{0}=-a_{0}+\sqrt{\alpha} \operatorname{ch} \psi, p_{3}^{1}=\sqrt{\alpha} \operatorname{sh} \psi, \psi \text { real }, 0<\alpha<\varrho\right\} .
$$


Letting then $a_{0}$ tend to 0 , one obtains analyticity at all the real points $\left\{p_{3}^{0}<\sqrt{p_{3}^{2}+\varrho}\right\}$. This proves (10) in the case of a massive theory. It is left to the reader so inclined to check by explicit calculation the geometrical situation described above.

In the case $m=0$, we first go back to the E.o.W. problem $T_{\varrho}^{+} \cup T_{\varrho}^{-} \cup C \bar{V}_{+}$which had furnished us with the complex neighbourhood $N_{+}$of $C \bar{V}_{+}$. By an application of the local Re-entrant Nose Theorem it can be easily seen that the region of coincidence $C \bar{V}_{+}$gets increased to a strictly larger open real set $R_{+}$; in particular a bit of the tip (the "nose") of the cone $\bar{V}_{+}$gets chopped off. If we now feed this qualitative information into the E.o.W. problem governing the analyticity domain of the "symmetric" respectively "antisymmetric" functions $F^{s}$ respectively $F^{a}$, we are led to compute the envelope of holomorphy $H$ of $T^{+} \cup T^{-} \cup\left(R_{+} \cap R_{-}\right)$, where $R_{-}=-R_{+}$. But $H$ contains as a subdomain the domain $\mathrm{D}$, which is invariant under complex Lorentz transformations. Hence by a well-known theorem (cf. e.g. [6]) $H$ is also invariant under the complex Lorentz group; in particular $H$ contains the real points

$$
\bigcup_{\Lambda \in \mathscr{L}_{+}^{\uparrow}} \Lambda\left(R_{+} \cap R_{-}\right)
$$

and these are necessarily of the form $\left\{p_{3}^{2}<m^{2}\right\}$, where $m$ is a strictly positive constant (depending may be on $\varrho$ ). We have thus reduced the problem to that of a massive theory and the proof of Lemma 1 is complete.

\section{Proof of the Theorem}

We next turn to the definition of the space-integral of the density $\varrho^{2}$. This is a standard procedure and we therefore keep it brief. To begin with we define a derivation $\delta^{3}$ on the ${ }^{*}$-algebra $\mathfrak{A}_{0}$ of locally smeared polynomials in the basic field $\phi$

$$
\delta(A)=\int d^{3} x[\varrho(x), A] \text { for } A \in \mathfrak{A}_{0} .
$$

This expression is well defined on the common dense domain $\left\{A \Omega: A \in \mathfrak{H}_{0}\right\}$ of the operators in $\mathfrak{A}_{0}$ because the integration extends only over a finite region of space. Due to the fact that $\Omega$ is cyclic and separating for $\mathfrak{A}_{0}$ we may then define a linear operator $Q$ on $D(Q)=\left\{A \Omega: A \in \mathfrak{U}_{0}\right\}$ by

$$
Q \cdot A \Omega=\delta(A) \Omega .
$$

It follows from relation (1) of the introduction that $\left(\Omega, \delta\left(A^{\prime} A\right) \Omega\right)=0$ for all $A, A^{\prime} \in \mathfrak{A}_{0}$ and therefore

$$
\left(\Omega, A^{\prime} Q A \Omega\right)=\left(\Omega, A^{\prime} \delta(A) \Omega\right)=-\left(\Omega, \delta\left(A^{\prime}\right) A \Omega\right) .
$$

Thus $Q$ is a closable operator with a densely defined adjoint $Q^{*}$ and $D\left(Q^{*}\right) \supset D(Q)$. If in particular $\varrho$ is a hermitian density then $Q$ is a hermitian operator.

\footnotetext{
2 We refrain from using the term charge for this quantity because it need not be connected with a superselection rule in general. If $\varrho$ were the energy density for example, we would end up with the Hamiltonian
}

3 A derivation $\delta$ is a linear mapping which satisfies $\delta\left(A^{\prime} A\right)=\delta\left(A^{\prime}\right) A+A^{\prime} \delta(A)$ on its domain 
The time-translated operators $Q(t)=T(t) Q T(t)^{-1}$ are also defined on $D(Q)$ because this domain is stable under the action of $T(t)^{-1}$. It is furthermore obvious that

$$
Q(t) A \Omega=\delta_{t}(A) \Omega
$$

where $\delta_{t}$ is the derivation which one gets from (12) if one replaces $\varrho(x)$ by $\varrho(t, \boldsymbol{x})$. The following statement on $\delta_{t}$ is a simple consequence of the results in the preceding chapter and positivity.

Lemma 2. Let $A \in \mathfrak{A}_{0}$. Then the relation

$$
\left(d^{N} / d t^{N}\right) \delta_{t}(A(x))=0
$$

holds on the domain $D(Q)$ for some finite $N$. The number $N$ does not depend on $x \in \mathbb{R}^{4}$.

Proof. Since $\varrho$ and $A$ are locally smeared polynomials in the basic field $\phi$ it follows from temperateness and locality that the derivatives

$$
\left(d^{N} / d t^{N}\right) \delta_{t}(A(x))
$$

exist for all $N$ on $D(Q)$ in the strong topology and that they are elements of $\mathfrak{U}_{0}$. It is therefore sufficient to prove

$$
\left(d^{N} / d t^{N}\right) \delta_{t}(A(x)) \Omega=0
$$

because $\Omega$ is a separating vector for $\mathfrak{U}_{0}$. Using temperateness and locality another time one can show that the norm $\left\|\delta_{t}(A) \Omega\right\|$ is polynomially bounded in $t$. (See e.g. [13].) On the other hand one knows from Lemma 1 that $\left(\Omega, A^{\prime} \delta_{t}(A) \Omega\right)$ is a polynomial in $t$ if $A, A^{\prime} \in \mathfrak{U}_{0}$. But $\left|\left(\Omega, A^{\prime} \delta_{t}(A) \Omega\right)\right| \leqq\left\|\delta_{t}(A) \Omega\right\| \cdot\left\|A^{\prime *} \Omega\right\|$ and therefore the degree of this polynomial cannot exceed a certain number which depends only on $A$. Hence

$$
\left(d^{N} / d t^{N}\right)\left(\Omega, A^{\prime} \delta_{t}(A) \Omega\right)=0
$$

for some $N$ and arbitrary $A^{\prime} \in \mathfrak{U}_{0}$. This proves the lemma for $x=0$. For arbitrary $x=\left(x_{0}, \boldsymbol{x}\right)$ the statement is a simple consequence of the fact that $\delta_{t}(A(x)) \Omega=$ $T(x) \delta_{t-x_{0}}(A) \Omega$.

What remains to be done is to show that the number $N$ in the above lemma can be fixed independently of $A$. To verify this we must specify an operator $B \in \mathfrak{U}_{0}$ which generates (together with its translates) an irreducible set of operators. A suitable candidate is $B=\int d^{4} x h(x) \phi(x)$. Here the test function $h(x)$ has compact support and $\tilde{h}(p)$ has no zeros in momentum space ${ }^{4}$. It is the second property of $h$ which makes it possible to construct from each test function $\tilde{f}(p)$ with compact support in momentum space another test function

$$
\tilde{g}(p)=\tilde{f}(p) / \tilde{h}(p)
$$

such that $\int d^{4} x g(x) B(x)=\int d^{4} x f(x) \phi(x)$. But the test functions with compact support are dense in the Schwartz space $\mathscr{S}$ and since $\phi$ is tempered and irreducible, it is obvious that $B$ has the desired properties. Now we can prove

$4 \quad$ It may be inferred from [1] that such functions exist 
Lemma 3. There exists a number $N$ such that

$$
\left(d^{N} / d t^{N}\right) \delta_{t}(A)=0
$$

on the domain $D(Q)$ for all $A \in \mathfrak{A}_{0}$.

Proof. As a consequence of the properties of $\delta_{t}$ mentioned earlier the operator $Q(t)$ defined in relation (15) is arbitrarily often differentiable with respect to $t$ and the domain $D(Q)$ is stable under the action of the derivatives $\left(d^{N} / d t^{N}\right) Q(t)$. Furthermore, $\delta_{t}(A)=[Q(t), A]$ for all $A \in \mathfrak{A}_{0}$. Bearing in mind Lemma 2 one gets therefore for some finite $N$ and arbitrary $x \in \mathbb{R}^{4}$

$$
\left[\left(d^{N} / d t^{N}\right) Q(t), B(x)\right]=\left(d^{N} / d t^{N}\right) \delta_{t}(B(x))=0
$$

where $B$ is the operator specified above. Thus $\left(d^{N} / d t^{N}\right) Q(t)$ commutes with an irreducible set of operators. It must therefore be a multiple of the identity which can only be zero because $Q(t) \Omega=\delta_{t}(1) \Omega=0$. But this implies

$$
\left(d^{N} / d t^{N}\right) \delta_{t}(A)=0
$$

for arbitrary $A \in \mathfrak{Q}_{0}$.

In the course of the above argument we incidentally proved the first part of our main theorem, viz.

$$
\left(d^{N} / d t^{N}\right) Q(t)=0
$$

for some finite $N$. As was indicated in the introduction we do not know in general for which values of $N$ this relation holds. (To answer this question one has probably to take also non-linear properties of the three-point function into consideration in the analysis of Chapter 2.) However we have a simple argument which shows that $N=1$ in all models with short range forces and a complete particle interpretation.

To begin with we have to make a brief excursion to collision theory ${ }^{5}$. We consider for simplicity the model of only one neutral particle with mass $m$. In such a model one can specify smeared polynomials $B$ in the basic field $\phi$ which create one-particle states from the vacuum $\Omega$. Furthermore, these operators can be chosen in such a way that $B^{*} \Omega=0$. We then define

$$
B_{f}(t)=\int d^{4} p \tilde{f}(p) e^{i t\left(p^{0}-\omega_{p}\right)} \tilde{B}(p)
$$

( $f$ being an arbitrary test function and $\omega_{\boldsymbol{p}}=\sqrt{\boldsymbol{p}^{2}+m^{2}}$ ) and construct the HaagRuelle approximants

$$
\Psi_{f_{1} \ldots f_{n}}(t)=B_{f_{1}}(t) \ldots B_{f_{n}}(t) \Omega .
$$

These sequences converge strongly in the limit of large $t$

$$
\text { s- } \lim _{t \rightarrow \infty} \Psi_{f_{1} \ldots f_{n}}(\dot{t})=\Psi_{f_{1} \ldots f_{n}}^{\text {out }}
$$

and the linear span of the vacuum $\Omega$ and the limit vectors $\Psi_{f_{1} \ldots f_{n}}^{\text {out }}$ is dense in the Hilbert space $\mathscr{H}$ if the model is asymptotically complete. Now we are prepared to prove

5 For detailed explanations and proofs of the statements made in the subsequent paragraph we refer to the lecture notes of Hepp [13] and the book of Jost [6] 
Lemma 4. In an asymptotically complete theory of a massive, neutral particle the relation

$$
\delta_{t}(A)=\delta(A)
$$

holds for all $A \in \mathfrak{A}_{0}$. Consequently

$$
Q(t)=Q \text {. }
$$

Proof. Owing to the mass gap, the Wightman functions of the basic field $\phi$ have strong clustering properties for large space like separations of their arguments. This makes it possible to extend $\delta_{t}$ from the algebra $\mathfrak{A}_{0}$ of locally smeared polynomials in $\phi$ to the algebra $\mathfrak{A}$ of all smeared polynomials in $\phi$. The extension is defined by

$$
\delta_{t}(A)=\operatorname{s-lim} \int_{R \rightarrow \infty} d_{|x| \leqq R} d^{3} x[\varrho(t, \boldsymbol{x}), A], \quad A \in \mathfrak{A}
$$

and the limit exists on the dense set of vectors $\{A \Omega: A \in \mathfrak{A}\}^{6}$. It follows then from (1) that $\left(A^{\prime} \Omega, \delta_{t}(A) \Omega\right)=-\left(\overline{\delta_{t}}\left(A^{\prime}\right) \Omega, A \Omega\right)$ for arbitrary $A, A^{\prime} \in \mathfrak{A}$ where $\overline{\delta_{t}}$ is given by relation (18) if one replaces $\varrho$ by $\varrho^{*}$. Since the operators $B_{f}(t)$ introduced above are elements of $\mathfrak{U}$ one gets

$$
\begin{aligned}
\left|\left(\Psi_{f_{1} \ldots f_{n}}(t), \delta_{t}(A) \Omega\right)\right| & =\left|\left(B_{f_{1}}(t) \ldots B_{f_{n}}(t) \Omega, \delta_{t}(A) \Omega\right)\right| \\
& =\left|\left(\bar{\delta}_{t}\left(B_{f_{1}}(t) \ldots B_{f_{n}}(t)\right) \Omega, A \Omega\right)\right| \\
& \leqq\left\|\bar{\delta}_{t}\left(B_{f_{1}}(t) \ldots B_{f_{n}}(t)\right) \Omega\right\| \cdot\|A \Omega\| \\
& \leqq c \cdot\|A \Omega\|
\end{aligned}
$$

and the constant $c$ does not depend on $t$. The last inequality can be verified using similar techniques as in the well known proof establishing the existence of collision states $[6,13]$. In particular one has to exploit the clustering properties of the Wightman functions and the asymptotic behaviour of solutions of the KleinGordon equation. Since the argument is straightforward we omit the details. Consider now the vector valued function $t \rightarrow \delta_{t}(A) \Omega$ with $A \in \mathfrak{A}_{0}$. It follows from Lemma 2 that this function is a polynomial of a certain degree $M$ in $t$

$$
\delta_{t}(A) \Omega=\sum_{n=0}^{M} t^{n} \cdot \Phi_{n} .
$$

The asymptotic completeness of the model guarantees that $\left(\Psi_{f_{1} \ldots f_{n}}^{\text {out }}, \Phi_{M}\right) \neq 0$ for some collision state $\Psi_{f_{1} \ldots f_{n}}^{\text {out }}$ if $\Phi_{M} \neq 0$. Therefore

$$
\left|\left(\Psi_{f_{1} \ldots f_{n}}^{\text {out }}, \delta_{t}(A) \Omega\right)\right| \geqq c_{1} \cdot|t|^{M}, \quad c_{1}>0
$$

for large enough $|t|$. On the other hand it follows from relations (19) and (20) that for certain constants $c_{2}, c_{3}$

$$
\begin{aligned}
& \left|\left(\Psi_{f_{1} \ldots f_{n}}^{\text {out }}, \delta_{t}(A) \Omega\right)\right| \\
& \leqq\left|\left(\Psi_{f_{1} \ldots f_{n}}(t), \delta_{t}(A) \Omega\right)\right|+\left|\left(\Psi_{f_{1} \ldots f_{n}}^{\text {out }}-\Psi_{f_{1} \ldots f_{n}}(t), \delta_{t}(A) \Omega\right)\right| \\
& \leqq c_{2}+c_{3} \cdot\left(1+|t|^{M}\right) \cdot\left\|\Psi_{f_{1} \ldots f_{n}}^{\text {out }}-\Psi_{f_{1} \ldots f_{n}}(t)\right\| .
\end{aligned}
$$

$6 \quad$ It should be noticed that $\delta_{t}$ does not map $\mathfrak{U}$ into itself in general 
But this is impossible if $M \neq 0$ because $\left\|\Psi_{f_{1} \ldots f_{n}}^{\text {out }}-\Psi_{f_{1} \ldots f_{n}}(t)\right\|$ approaches zero for large positive $t$. Therefore $\delta_{t}(A) \Omega=\delta(A) \Omega$ for all $t$ and since $\Omega$ is separating for $\mathfrak{U}_{0}$ the statement is proved.

\section{Concluding Remarks}

We conclude this paper with a list of generalizations of the main theorem which are more or less obvious:

a) It was of no relevance in our discussion that space has dimension $d=3$. The analysis can be carried through for any $d \geqq 1$ and the theorem holds in a model world with an arbitrary number of spatial dimensions.

b) The assumption that $\varrho$ is a polynomial in the basic field $\phi$ can be relaxed. It is only needed in the proofs that $\varrho$ is a local polynomial in temperate fields which are elements of the Borchers class of $\phi$. Therefore our theorem covers also the physically relevant examples of tensor currents treated in Ref. [3] and in the articles quoted there.

c) Wightman theories with an arbitrary (but finite) number of fundamental Bose and Fermi fields do not cause any additional difficulties and the theorem holds equally well in these models. For infinitely many basic fields the proof of Lemma 3 is no longer applicable. (It was crucial there that one can specify a finite number of fields which generate an irreducible set of operators.) However, Lemmas 1, 2 and 4 remain true with obvious modifications.

d) For Haag-Araki fields [14] (although they are bounded operators) the argument in Chapter 2 cannot be simplified because it is based on linear properties of the vacuum expectation values, only. However, the proofs of Lemmas 2 and 3 (where positivity played an important role) can be reduced to some few lines: if $\varrho$ is a localized, bounded operator and $A$ a localized Haag-Araki field it follows simply from locality that

$$
\left\|\delta_{t}(A)\right\| \leqq \int d^{3} x\|[\varrho(t, \boldsymbol{x}), A]\| \leqq c \cdot\left(1+|t|^{3}\right) \cdot\|\varrho\|\|A\|
$$

and $c$ depends only on the localization regions of $\varrho$ and $A$. Thus $N \leqq 4$ in Lemma 3 . Therefore a slightly improved version of the main theorem holds in this framework of field theory.

e) Lemmas 1, 2 and 3 remain also valid for densities $\varrho[x]$ which are finite sums of localized operators $\varrho_{(m)}$ multiplied with monomials $x^{(m)}=x_{0}^{m_{0}} x_{1}^{m_{1}} x_{2}^{m_{2}} x_{3}^{m_{3}}$ :

$$
\varrho[x]=T(x)\left(\sum x^{(m)} \varrho_{(m)}\right) T(x)^{-1} .
$$

That our arguments also apply to such densities illustrates the fact that we did not fully exploit translational covariance in the proofs. However, translational covariance was essential for Lemma 4 and in particular for the proof of relation (19). This estimate has to be modified by

$$
\left|\left(\Psi_{f_{1} \ldots f_{n}}(t), \delta_{t}(A) \Omega\right)\right| \leqq c \cdot\left(1+|t|^{M}\right) \cdot\|A \Omega\|
$$

where $M$ is the highest degree of the monomials $x^{(m)}$ in $\varrho[x]$. Then

$$
\left(d^{N} / d t^{N}\right) Q[t]=0
$$

for some $N \leqq M+1$ and this result fits completely with intuitive expectations. 
Finally we want to point out that there exist densities $\varrho$ which are not expressible as finite sums of smeared tensor fields but which generate nontrivial constants of motion $Q$, nevertheless. $A$ simple example is

$$
\varrho=\int d^{4} x d^{4} y f(x, y): \phi(x) \phi(y) \text { : with } f(x, y)=\left[\sum_{\alpha=0}^{3} \frac{\partial}{\partial x_{\alpha}} \frac{\partial}{\partial y_{\alpha}}+m^{2}\right] g(x, y)
$$

where $\phi$ is a scalar, free field with mass $m$ and $g$ an arbitrary test function with compact support. It is easy to verify that the corresponding $Q$ 's are conserved and in general non-trivial. This is clearly due to the fact that there are infinitely many conservation laws in a free field theory. It is unlikely that similar multiply localized densities generate non-trivial $Q$ 's in the presence of interaction (see e.g. [15]). There the only genuine sources of constants of motion seem to be smeared tensor-currents.

Acknowledgements. We gratefully acknowledge stimulating discussions with H. Epstein and R. Haag.

\section{References}

1. Gal-Ezer,E., Reeh,H.: Charges as integrals over densities: An alternative formulation of Coleman's theorem. Commun. math. Phys. 43, 137 (1975)

2. Coleman, S.: The invariance of the vacuum is the invariance of the world. J. Math. Phys. 7, 787 (1966)

3. Gal-Ezer,E., Reeh,H.: Coleman and Coleman-Okubo theorems in relativistic quantum field theory. Fortschr. Phys. 22, 481 (1974)

4. Streater, R.F., Wightman, A. S.: PCT, Spin and statistics and all that (Benjamin, New York, 1964)

5. Källen, G., Wightman, A. S.: The analytic properties of the vacuum expectation value of a product of three scalar local fields. Dan. Vid. Selsk. Math. Fys., Skr. 1, No. 6 (1958)

6. Jost, R.: The general theory of quantized fields (American Mathematical Society, Providence, RI, 1965)

7. Epstein, H.: Some analytic properties of scattering amplitudes in quantum field theory, Proc. Brandeis Summer Institute, 1965. New York: Gordon and Breach 1966

8. Dyson, F. J.: Integral representations of causal commutators, Phys. Rev. 110, 1460 (1958); Stora, R.: Some applications of the J.L.D. theorem, Lecture notes in Math. 449. Berlin, Heidelberg, New York: Springer 1975

9. Bros, J., Epstein,H., Glaser, V., Stora, R.: Quelques aspects globaux des problèmes d'E.o.W., Lecture notes in Math. 449. Berlin, Heidelberg, New York: Springer 1975

10. Streater, B.F.: Special methods of analytic completion in field theory. Proc. Roy. Soc. A 256, 39 (1960). (See also R. Stora in Ref. [8])

11. Bros, J., Epstein, H., Glaser, V.: Some rigorous analytic properties of the four point function in momentum space. Nuovo Cimento 31, 1265 (1964)

12. Epstein,H., Glaser,V., Martin,A.: Polynomial behaviour of scattering amplitudes in theories with local observables. Commun. math. Phys. 13, 257 (1969)

13. Hepp, K.: The connection between Wightman and LSZ quantum field theory. Proc. Brandeis Summer Institute, 1965. New York: Gordon and Breach 1966

14. Araki, H., Haag, R.: Collision cross-sections in terms of local observables. Commun. math. Phys. 4, 77 (1967)

15. Coleman, S., Mandula, J.: All possible symmetries of the $S$-matrix. Phys. Rev. 159, 1251 (1967)

Communicated by R. Haag

Received January 13, 1976 